\title{
Deep Learning Classification of Building Types in Northern Cyprus
}

\author{
Mubarak Ahmad Muhammad \\ Dept. of Electrical and Electronics Engineering \\ Near East University \\ Nicosia, Cyprus \\ Email: mdanfulani [AT] gmail.com
}

\author{
Sertan Serte \\ Dept. of Electrical and Electronics Engineering \\ Near East University \\ Nicosia, Cyprus \\ Email: sertan.serte [AT] neu.edu.tr
}

Abstract - Among the areas where AI studies centered on developing models that provide real-time solutions for the real estate industry are real estate price forecasting, building age, and types and design of the building (villa, apartment, floor number). Nevertheless, within the ML sector, DL is an emerging region with an Interest increases every year. As a result, a growing number of DL research are in conferences and papers, models for real estate have begun to emerge. In this study, we present a deep learning method for classification of houses in Northern Cyprus using Convolutional neural network.

This work proposes the use of Convolutional neural networks in the classification of houses images. The classification will be based on the house age, house price, number of floors in the house, house type i.e. Villa and Apartment.

The first category is Villa versus Apartments class; based on the training dataset of 362 images the class result shows the overall accuracy of $96.40 \%$. The second category is split into two classes according to age of the buildings, namely 0 to 5 years Apartments 6 to 10 years Apartments. This class is to classify the building based on their age and the result shows the accuracy of $87.42 \%$. The third category is villa with roof versus Villa without roof apartments class which also shows the overall accuracy of $87.60 \%$. The fourth category is Villa Price from 10,000 euro to 200,000 Versus Villa Price from 200,000 Euro to above and the result shows the accuracy of $81.84 \%$. The last category consists of three classes namely 2 floor Apartment versus 3 floor Apartment, 2 floor Apartment versus 4 floor Apartment and 2 floor Apartment versus 5 floor Apartment which all shows the accuracy of $83.54 \%$, $82.48 \%$ and $84.77 \%$ respectively.

From the experiments carried out in this thesis and the results obtained we conclude that the main aims and objectives of this thesis which is to used Deep learning in Classification and detection of houses in Northern Cyprus and to test the performance of AlexNet for houses classification was successful. This study will be very significant in creation of smart cities and digitization of real estate sector as the world embrace the used of the vast power of Artificial Intelligence, machine learning and machine vision.

Keyword: Deep learning; AlexNet; Neural Network; Northern Cyprus; Apartment; Villa, Real Estate

\section{INTRODUCTION}

For each economy in the world, the importance of real estate statistics is undisputed and widely accepted. The drivers of demand and the availability of prices for real estate vary across nations. Such drivers depend on various variables that can alter over time. The real estate market is, indeed, competitive and it can be a challenge to recognize a complete list of drivers. However, it may not be practical for researchers to include an extensive description of factors.[1].

Among the areas where AI studies centered on developing models that provide real-time solutions for the real estate industry are real estate price forecasting, building age, and types and design of the building (villa, apartment, floor number). Nevertheless, within the ML sector, DL is an emerging region with an Interest increases every year. As a result, a growing number of DL research are in conferences and papers, models for real estate have begun to emerge. In this study, our emphasis is to provide an approach using deep learning for classification of houses in Northern Cyprus.

As a machine learning technique, deep learning emulates the function of the human brain after analyzing the given datasets to make a human-like decision. Deep learning is an algorithm that uses artificial multi-layer neural networks to learn the characteristics of unstructured data correlation. It retrieves the functionality using raw data in the form of regression and or classification[2].

In order to increase the level of computer vision, the use of the deep learning approach of the Convolutional neural network in image classification is praised worldwide. Using training algorithms, deep learning can identify the input datasets and allow the computer to classify or identify the image depending on the form of training.

This work proposes the use of Convolutional Neural Networks in building types classification using houses images. The classification will be based on the house age, house price, number of floors in the house, house type i.e. Villa and Apartment. For this reason, database of the houses images is collected and going to be used with our system.

\section{RELATED WORK}

Although the building age, price and types of the building are significant parameter in the construction specifications, the information is not always accessible or complete. For the 
classification of the building type or detection of buildings age, few researches have been performed.

Along with other research efforts, Henn et al introduces a Support Vector Machines (SVMs) classifier, an advanced machine learning tool for semantic enrichment of coarse 3D city models by deriving the form of construction[3].

Henn et al research automatically define the form of construction In LOD1 models with a few building features, e.g. floors number and many attributes of the local background e.g. distance to the nearest school using supervised learning (support vector machines SVM)[3].

Supervised machine learning methods of SVM are used for the selection of the most probable roof model. Unlike standard approaches, supervised classification is capable of integrating additional features that allow a substantial improvement in the accuracy of model selection[4]

Yan Li et al[5] The method was introduced and evaluated by a new approach to direct estimation of building age from Google Street View images. The algorithm consists of three main steps: downloading Google Street View images, extracting image characteristics, and estimating the age of the house. Results for the North and West Metropolitan Region of Victoria indicate that construction age estimation can be accurately forecast with

DCNN. In the research, they used different CNN Architectures which include DenseNet161, AlexNet, ResNet 18 and ResNet 50. Among them, DenseNet161 shows the best efficiency, and it confirms that the deeper CNN structure appears to produce more detailed results[5].

F. Biljeck et al[6] The paper discusses the possibilities for Forecasting the construction age of buildings using random forest regression from other attributes. The purpose of the research is to find whether the age of buildings or the year of construction using $3 \mathrm{D}$ GIS and machine learning can be inferred.
Deep learning Researchers have also used conventional neural networks to classify images and produce a good result.

Using Fundus images, Sertan Serte and Ali Serenar [7] presents a generalized deep learning model for glaucoma detection. The model is trained and evaluated on several datasets and architectures, contrary to previous studies. The findings show that $80 \%$ of the time, the model is equal or better than previous work in the literature. They [8] also introduces, using fundus images to detect early and advanced glaucoma automatically. The ResNet-50 and GoogleNet CNN algorithms are trained and fine-tuned using transfer learning to classify. The suggested method also produces good result.

\section{STUDY AREA}

Northern Cyprus comprises of the north east portion of the Cyprus island. Turkish Republic of Northern Cyprus is its official name. The island only recognized by Turkey but international communities still considered both North and South to be one country[9].

TRNC ranges from Morpho Bay to the west and to the top of the northeastern Karpass Peninsula. The village of Louroujina is its southernmost point. A United Nations-controlled buffer zone extends between South and North and separates the capital Nicosia on both parts[10].

In recent years, Northern Cyprus has experienced a remarkable construction boom. These building activities have increased dramatically following the failed peace plan initiated in 2003 by former UN Secretary-General Kofi Annan[12]. Ever since, Northern Cyprus's architectural design has shown a strong tendency towards post-moderation. The creation of Northern Cyprus' modern architecture is inspired from improvements since about the 1980s in Turkey and by Turkish designers who have not been officially recognized for their job. The buildings types in Northern Cyprus are mostly Villa, residence or apartment, penthouse, bungalow[13]. 


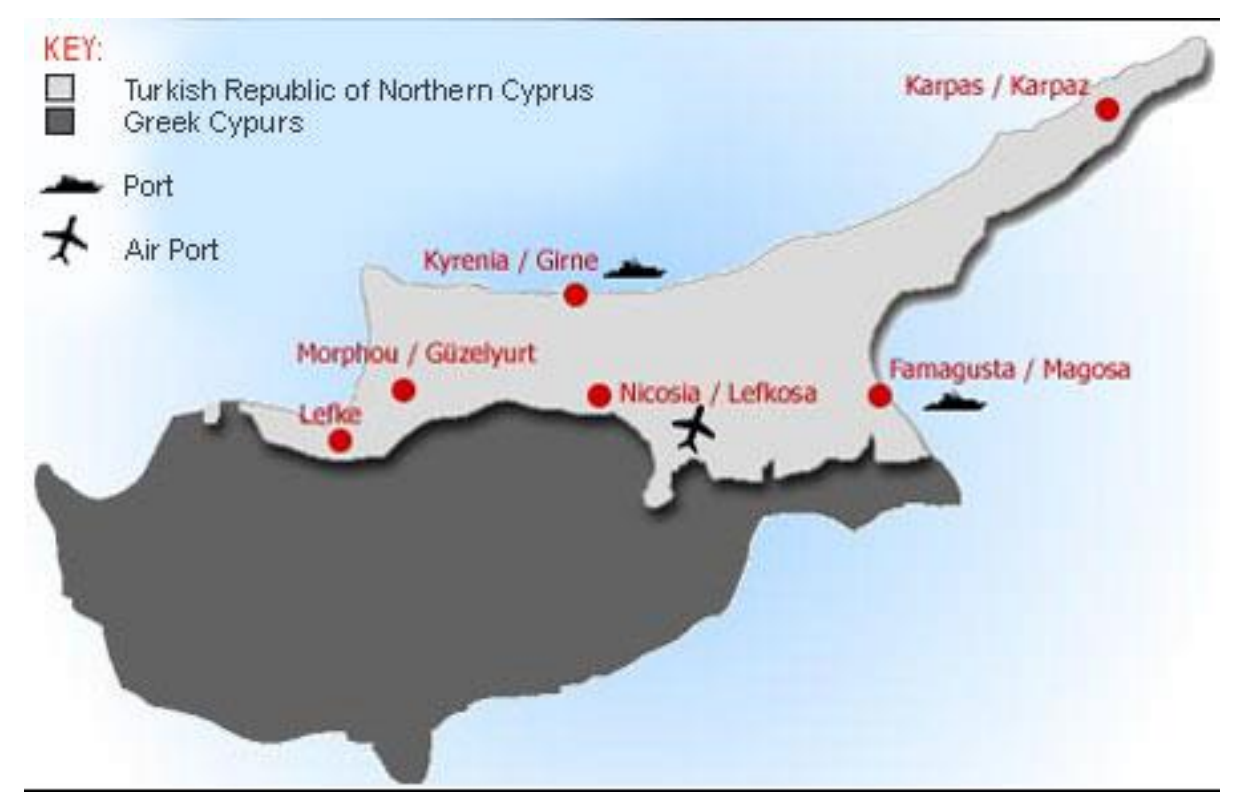

Figure 1: Map of Northern Cyprus[11]

\section{DATASET}

The beginning of this work is dataset collection, all image was access from the popular Northern Cyprus real estate company 101 Elver. The company is known as the leading re al estate portal of Northern Cyprus[14]. Their mission is to create a platform where all properties for sale or rent in Cyprus are located, where buyers and sellers meet. All the images in the data set are collected from Nicosia Area of Northern Cyprus. The dataset can be access at www.101elver.com.
The first category is Villa versus Apartments class. The second category is split into two class namely 0 to 5 years old Apartments 6 to 10 years old Apartments. The third category is villa with roof versus Villa without roof apartments class. The fourth category is Villa Price from 10,000 euro to 200,000 Versus Villa Price from 200,000 Euro to above. The last category consists of three classes namely 2 floor Apartment versus 3 floor Apartment, 2 floor Apartment versus 4 floor Apartment and 2 floor Apartment versus 5 floor Apartment.

Table 1: Classes of the houses and the number of images in each case

\begin{tabular}{llll}
\hline Class & $\begin{array}{l}\text { Number } \\
\text { images }\end{array}$ & $\begin{array}{c}\text { of } \\
\text { Training } \\
\text { Image }\end{array}$ & $\begin{array}{l}\text { Testing } \\
\text { Image }\end{array}$ \\
\hline & & & 110 \\
Apartment & 219 & 110 & 72 \\
Villa & 143 & 72 & 63 \\
0-5 Years old Apartments & 207 & 145 & 44 \\
6-10 Years old apartments & 145 & 101 & 63 \\
Villa with roof & 209 & 146 & 59 \\
Villa without roof & 195 & 137 & 62 \\
Villa Price from 10,000 Euro to 200,000 Euro & 206 & 144 & 90 \\
Villa Price from 200,000 Euro to Above & 301 & 211 & 51 \\
Apartments with 2 floors & 170 & 119 & 45 \\
Apartments with 3 floors & 151 & 106 & 31 \\
Apartments with 4 floors & 104 & 73 & 40 \\
Apartments with 5 floors & 132 & 92 & \\
Total & & & \\
\hline
\end{tabular}




\section{METHOD}

\section{A. Preprocessing}

The dataset has been arranged five different categories each one contain sub folders of the classes to be classify. The name of each sub folder is the name of the class. To take care of data inadequacy, we used Data augmentation techniques[15][16]. Also, the method supplies the network with the required details of learning of training data and reduced the probability of over fitting. We have images of various sizes in the dataset, but the AlexNet architecture needs 256X256 input images[15]. We have therefore changed the training and testing of images using online bulk resizer https://bulkresizephotos.com/en to render images appropriate for our network Some additional augmentation operations were added, such as randomly rotating the training images along the vertical axis and converting images up to 30 pixels in both vertically and horizontal[17]. MATLAB's image Data Augmenter library has been used for all the preprocessing tasks, which have various sets of data pre - processing options, such as translation, reflection, scaling, rotation, scaling, augmentation and shearing have been used in the training results[18][19].

\section{B. Alexnet}

Against all traditional machine learning and computer vision methods, AlexNet achieved state-of-the-art detection performance. It was an exciting development for object recognition and classification tasks in the field of machine learning and computer vision and is the point in history from which interest in deep learning has rapidly grown[20].
Compared to LeNet, Alex Krizhevesky and others introduced this deeper and wider CNN model in 2012 and won the ImageNet Large Scale Visual Recognition Challenge (ILSVRC), the most difficult ImageNet challenge for object detection[20].

Our network architecture is described in the figure below. It comprises eight layers that have been learned, five that are convolutional and three that are fully connected. Some of the new or unique features of the architecture of our network are listed below.

The first five are convolutional layer and the remaining three are completely connected. Convolution and Max-Pooling with LRN (Local Response Normalization) are done by the first convolutional layer, in which ninety-six 11 x 11 in size different receptive filters are used. The Max-Pooling operations are conducted with $3 \times 3$ filters with a step size of 2[21].

The $2^{\text {nd }}, 4$ th and $5^{\text {th }}$ convolution layer kernels are only linked to the kernel maps on the preceding layer that are on the same GPU. The $3^{\text {rd }}$ convolution layer kernels are linked to kernel maps on $2^{\text {nd }}$ layer.

Max-pooling layers follow all feedback layers in the preceding layer and the $5^{\text {th }}$ convolutional layer is linked to all neurons by the neurons in the fully connected layers. The ReLU is added to the output of fully-connected and convolutional layer[15].

An input image is filtered by the $1^{\text {st }}$ convolutional layer of $224 \times 224 \times 3$ with ninety-six kernels with size with a distance between adjacent neurons' receptive field centers of 4-pixel kernel map.

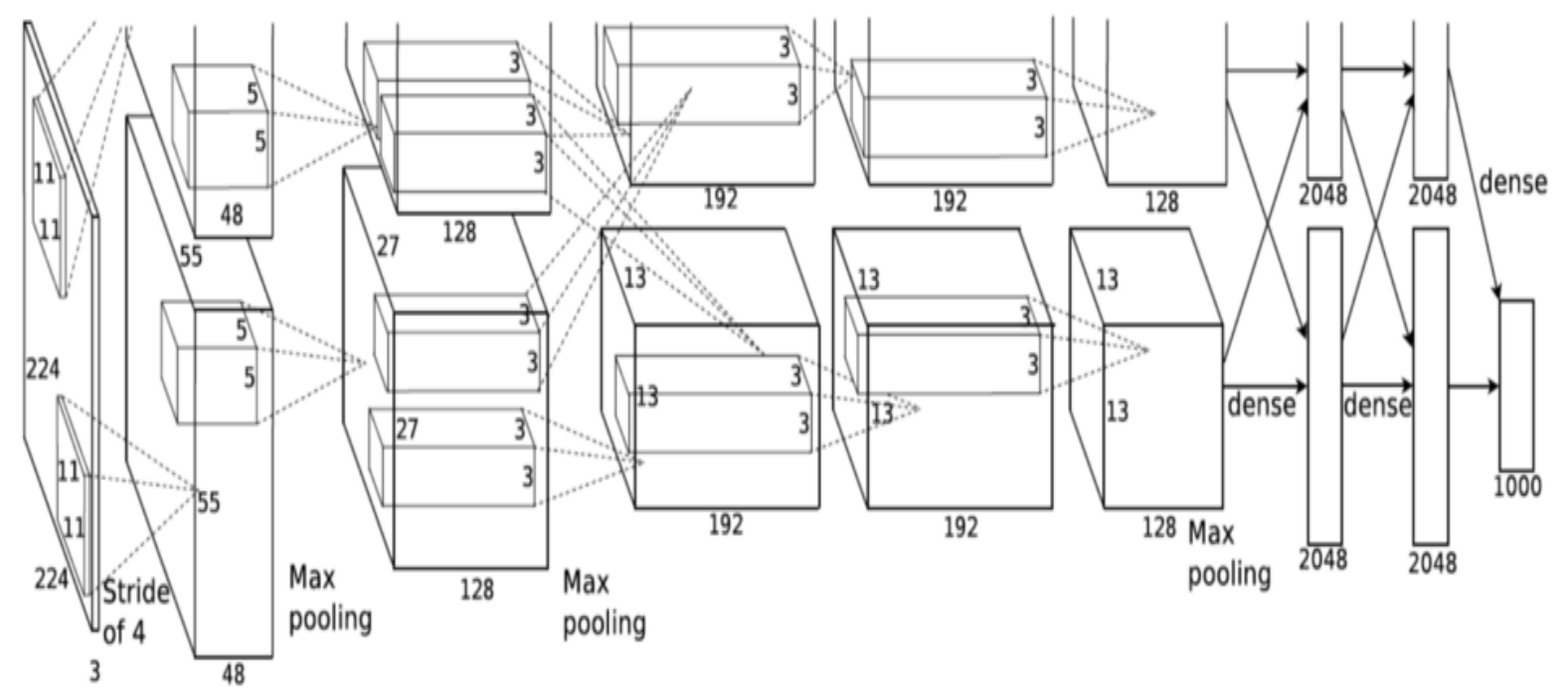

Figure 2: AlexNet Architecture[22] 
The 2nd convolutional layer takes output of the first convolutional layer as input and filters it with 256 kernels of 5 x 5 x 48 size. Without any interference of pooling or normalization layer, $3^{\text {rd }}, 4^{\text {th }}$ and $5^{\text {th }}$ convolutional layers are related[23].

The $3^{\text {rd }}$ convolutional layer of size $3 \times 3 \times 256$ has 384 kernels connected to the outputs of the $2^{\text {nd }}$ convolutional layer. The $4^{\text {th }}$ convolutional layer of size $3 \times 3 \times 192$ has 384 kernels, and the $5^{\text {th }}$ convolutional layer of size $3 \times 3 \times 192$ has 256 kernels. Which makes the fully-connected layers to have the total of 4096 neurons each[24]

\section{Learning details}

For our $1^{\text {ST }}$ Model (0-5 years vs. 5-10 years old), we train our model with weighted learning rate of 10 , mini batch size 10 , MaxEpoch 100 and Valid frequency of 3. We used $70 \%$ of our data for training and $30 \%$ for validation.

For the $2^{\text {nd }}$ models (Villa with roof vs. Villa without roof, Villa Price from 10,000 Euro to 200,000 Euro Vs. Villa Price from 200,000 Euro to Above and 2 floor Apartments Vs. 3,4and 5 floor Apartment) we train them with weighted learning rate of 20, mini batch size 20, MaxEpoch 100 and Valid frequency of 3 . We used $70 \%$ of our data for training and $30 \%$ for validation.

We train $3^{\text {rd }}$ first model (Villa Vs. Apartment) with weighted learning rate of 25, basic learning rate of 25, Minibatch size of 10, MaxEpochs of 100, valid frequency 3.

We also used $50 \%$ of our data for training and $50 \%$ for Validation shown in table below.

For all the models, all the network parameters used was adjusted manually throughout training to get the best result. For all the models, all the network parameters used was adjusted manually throughout training to get the best result.

MATLAB Version 2019a was used for implementation. The network was developed on a personal computer with $7^{\text {th }}$ Gen intel(R) Core i7-7500u and NVIDIA ${ }^{\circledR}$ GeForce 940MX SUPER GPU*1 MATLAB version R2019a was used.

\section{Performance Matrices}

Accuracy is a data measure described as the proportion of data samples correctly classified from the result to all samples. specificity and sensitivity similarly, are Statistical indicators that accurately forecast the percentage of Both the tests with positive data and a part of all the negative data samples respectively[25].

For of class of input images, True Negative (TN), True positive (TP), False Negative (FN) and False Positive (FP), result obtained may vary according to result obtained from the confusion matrix. If an apartment is forecast as an apartment, it shows a TP for that class. In contrast to this class, all the accurate predictions for other classes are referred to as TN. If an input image of an apartment is predicted as a villa, it is considered false positive[26].

In a nut shell Sensitivity tests the proportion of accurately labeled data correctly classify to the overall data correctly identified as positive classification[27]. Specificity tests how good the other groups are estimated by the algorithm. Accuracy tests the overall accuracy of the algorithm's classification rate[25].

\section{RESULT}

\section{A. Villa and Apartments Class}

Transfer learning with pre-trained AlexNet was used in the system proposed. From the result we obtained, we can believe that there is a great success was achieved by the proposed approach where the Villa vs. Apartment class is precisely classified. The accuracy overall achieved is $96.40 \%$ as shown from the figure below, we can see that the values of other metrics are Sensitivity is $98.17 \%$, Specificity is $97.36 \%$, Prevalence is $98.20 \%$ Positive Predicted Value is $98.37 \%$ and Negative Predicted Value is $96.21 \%$. The result is summarized in table below

\section{B. 0-5 Years and 6-10 years (old) Apartments Class}

On the basis of the results obtained, we may conclude that a good success was achieved by the proposed approach where the classification of 0-5 Years Vs. 6-10 years age Apartments Class is $87.42 \%$ accurate as we shown from the figure below. we can see that the values of other metrics are Sensitivity is $85.29 \%$, Specificity is $80.68 \%$, Prevalence is $89.20 \%$ Positive Predicted Value is $88.17 \%$ and Negative Predicted Value is $81.67 \%$. The result is summarized in table below

The training cycle parameters are also recorded such as Epoch, number of iteration and iteration per Epoch and validation frequency as also recorded.

\section{Villa With roof and Villa without roof class}

The accuracy overall achieved is $87.60 \%$ as shown from the figure below, we can see that the values of other metrics are Sensitivity is $93.65 \%$, Specificity is $81.03 \%$, Prevalence is $59.21 \%$ Positive Predicted Value is $82 \%$ and Negative Predicted Value is $82.96 \%$. The result is summarized in table below

\section{Villa Price Class}

The accuracy achieved as was $81.84 \%$. The values of other metrics are sensitivity of $80.56 \%$ which shows the proportion of accurately labeled data correctly identified to the overall number of data correctly identified as positive is not is reliable. 
The value of specificity is recorded at $85.48 \%$ which is good performance. $79.29 \%, 72.96 \%$ and $82 \%$ for prevalence, positive predicted value and Negative predicted Value respectively. The result is summarized in table below.

On the basis of the results obtained, the proposed method is needed to be improving to achieve good result.

\section{E. 2 floors and 3 floors Apartment Class}

The overall accuracy achieved was $83.84 \%$. The values of other metrics are sensitivity of $84.31 \%$ which shows the result of the proportion of accurately labeled data correctly identified to the overall number of data correctly identified as positive is good.

The value of specificity is recorded at $80 \%$. The result shows how the other groups are estimated by the algorithm is good. $83.13 \%, 69.23 \%$ and $61.43 \%$ for prevalence, positive predicted value and Negative predicted Value respectively. The result is summarized in table below.

On the basis of the results obtained, the proposed method is needed to be improving to achieve good result.

\section{F. 2 floors versus 4 floors Apartment class.}

Based on the result obtained, we may conclude that a great success was achieved by the proposed approach where the 2 floor and 4 floors Apartment class is precisely classified.

Overall accuracy was $82.48 \%$. The values of other metrics are $89.41 \%$ for sensitivity, $71.15 \%$ for Specificity, $62.04 \%$ for prevalence, $80.43 \%$ for positive predicted value and $83.52 \%$ for Negative predicted Value. the result is summarized in table below.

\section{G. 2 floors and 5 floor Apartments Class}

The accuracy was recorded at $84.77 \%$. The values of other metrics are $87.06 \%$ for sensitivity, $81.82 \%$ for specificity, $56.29 \%$ for prevalence, $83.08 \%$ for positive predicted value and 86.05 .

the result is summarized in table below. Hence the proposed system performance is good.

Table 2. Table of results

\begin{tabular}{|c|c|c|c|c|c|c|c|}
\hline Dataset & Class & $\begin{array}{l}\text { Predictive } \\
\text { Positive } \\
\text { Value }\end{array}$ & $\begin{array}{l}\text { Predictive } \\
\text { Negative } \\
\text { Value }\end{array}$ & Prevalence & $\begin{array}{l}\text { Accuracy } \\
\%\end{array}$ & $\begin{array}{l}\text { Sensitivity } \\
\%\end{array}$ & $\begin{array}{l}\text { Specificity } \\
\%\end{array}$ \\
\hline $\begin{array}{l}\text { Category } \\
\text { One }\end{array}$ & $\begin{array}{l}\text { Apartments } \\
\text { Villa }\end{array}$ & 0.9837 & 0.9621 & 0.9820 & 96.40 & 98.17 & 97.36 \\
\hline $\begin{array}{l}\text { Category } \\
\text { Two }\end{array}$ & $\begin{array}{l}\text { 0-5 Years apartments } \\
\text { Vs } \\
\text { 6-10 Years Apartments }\end{array}$ & 0.8817 & 0.8167 & 0.8920 & 87.42 & 85.29 & 80.68 \\
\hline $\begin{array}{l}\text { Category } \\
\text { Three }\end{array}$ & $\begin{array}{l}\text { Villa with roof Vs } \\
\text { Villa without roof }\end{array}$ & 0.8429 & 0.9216 & 0.5207 & 87.60 & 93.65 & 81.03 \\
\hline $\begin{array}{l}\text { Category } \\
\text { Four }\end{array}$ & $\begin{array}{l}\text { Villa Price from } 10,000 \text { euro to } \\
200,000 \text { Vs } \\
\text { Villa Price from } 200,000 \text { Euro } \\
\text { to above }\end{array}$ & 0.8200 & 0.5296 & 0.5929 & 81.84 & 85.56 & 85.48 \\
\hline \multirow{3}{*}{$\begin{array}{l}\text { Category } \\
\text { Five }\end{array}$} & 2 floor Vs 3 floor Apartments & 0.6143 & 0.6923 & 0.5313 & 83.54 & 84.31 & 80.00 \\
\hline & 2 floor Vs 4 floor Apartment & 0.8352 & 0.8043 & 0.6204 & 82.48 & 89.41 & 71.15 \\
\hline & 2 floor Vs 5 floor Apartment & 0.8605 & 0.8308 & 0.5629 & 84.77 & 87.06 & 81.82 \\
\hline
\end{tabular}




\section{CONCLUSION AND RECOMMENDATIONS}

In this paper, we review Artificial Neural Networks, its working principles and training algorithm such as back propagation learning techniques. Deep learning was discussed which include deep learning approaches such supervised learning, semi-supervised, un-supervised and reinforcement learning. Convolutional neural network has a significant effect on image classification[15][28] that makes it suitable candidate to use in this research.

After study and implementation of deep learning in the field of house classification it is concluded that deep leaning while using AlexNet provide very good result. First these images were downloaded from 101elever, a leading real estate company in Cyprus and the image is resized to fit our network[29].

The first category is Villa versus Apartments class; based on the training dataset of 362 images the class result shows the overall accuracy of $96.40 \%$. This shows that villa vs. apartment class has been very well classified. The second category is villa with roof versus Villa without roof apartment's class which also shows the overall accuracy of $87.60 \%$.

The first category is split into two classes namely 0 to 5 years Apartments 6 to 10 years Apartments. This class is to classify the building based on their age and the result shows the accuracy of $87.42 \%$.

The fourth category is Villa Price class from 10,000 euro to 200,000 Versus Villa Price from 200,000 Euro to above and the result shows the accuracy of $81.84 \%$

The last category consists of three classes namely 2 floors Apartment versus 3 floors Apartment, 2 floors Apartment versus 4 floors Apartment and 2 floors.

Apartment versus 5 floor Apartment which all shows the accuracy of $83.54 \%, 82.48 \%$ and $84.77 \%$ respectively.

From the experiments carried out in this research and the results obtained we conclude that the main aims and objectives of this research which is to used Deep learning in Classification and detection of houses in Northern Cyprus and to test the performance of AlexNet for houses classification was successful.

This study will be very significant in creation of smart cities and digitization of real estate sector as the world embrace the used of the vast power of Artificial Intelligence, machine learning, image processing and machine vision[5][30].

Future work will focus on the extension of building types classes and on the consideration of additional or more differentiated infrastructural features such villa versus bungalow, house with swimming pool and house without swimming pool, more precise building age, more detail building price, and so on.

Also, for the CNN architecture, we can test our proposed networks using other CNN architecture like ResNet, GoogleNet and DenseNet

\section{REFERENCES}

[1] G. Gilani, “ASSESSING FLEXIBILITY IN REAL ESTATE MASS HOUSING," 2020, doi: 10.4013/arq.2020.161.09.

[2] K. Arulkumaran, M. P. Deisenroth, M. Brundage, and A. A. Bharath, "A Brief Survey of Deep Reinforcement Learning," pp. 1-16.

[3] A. Henn, C. Römer, and G. Gröger, "Automatic classification of building types in 3D city models Using SVMs for semantic enrichment of low resolution building data," pp. 281-306, 2012, doi: 10.1007/s10707-011-0131-x.

[4] A. Henn, G. Gröger, V. Stroh, and L. Plümer, "ISPRS Journal of Photogrammetry and Remote Sensing Model driven reconstruction of roofs from sparse LIDAR point clouds," vol. 76, pp. 17-29, 2013, doi: 10.1016/j.isprsjprs.2012.11.004.

[5] Y. Li, Y. Chen, A. Rajabifard, K. Khoshelham, and M. Aleksandrov, "Estimating Building Age from Google Street View Images Using Deep Learning," no. 40, pp. 1-7.

[6] R. Sensing, S. I. Sciences, F. Biljecki, and M. Sindram, "ESTIMATING BUILDING AGE WITH 3D GIS," vol. IV, no. October, pp. 26-27, 2017.

[7] S. Serte, "A Generalized Deep Learning Model for Glaucoma Detection," 2019

[8] A. Serener, "Transfer Learning for Early and Advanced Glaucoma Detection with Convolutional Neural Networks," pp. 6-9.

[9] A. H. Robertson, Yearbook of the European Convention on Human Rights/Annuaire de la Convention Europeenne des Droits de L'Homme: The European Commission and European Court of Human Rights/Commission et Cour Europeennes des Droits de L'Homme. Springer, 2013.

[10] Ü. Alptekin and A. Ertalcs, "Kuzey K\{li\}br\{\i\}s Türk Cumhuriyeti'nde $1995 \quad \mathrm{y}\{\mathbf{i}\} 1\{\backslash \mathrm{i}\} \quad$ orman yang $\{\backslash \mathrm{i}\} \mathrm{n}\{\mathbf{i}\}$ sonras $\{\backslash \mathrm{i}\}$ ndaki $\quad \mathrm{a}\{\mathbf{\mathrm { u }}\{\mathrm{g}\}\}$ açland $\{\backslash \mathrm{i}\}$ rmalardan $\quad$ gözlemler,' ^.I\} stanbul Üniversitesi Orman Fakültesi Derg., vol. 43, no. 3-4, pp. 133-144, 1993.

[11] "North Cyprus Map."

[12] M. Kiessel, D. Yücel-besİm, and A. Tozan, "THE NEW ARCHITECTURAL CLASSICISM IN,” 2011, doi: 10.4305/METU.JFA.2011.2.8.

[13] A. Sivakumar, "Pelletized fly ash lightweight aggregate concrete: A promising material," J. Civ. Eng. Constr. Technol., vol. 3, no. 2, pp. 42-48, 2012, doi: 10.5897/jbd11.088.

[14] "101 Elver."

[15] A. Uçar, Y. Demir, and C. Güzeliş, "Object recognition and detection with deep learning for autonomous driving applications," Simulation, vol. 93, no. 9, pp. 759-769, 2017, doi: 10.1177/0037549717709932.

[16] A. Vyas, S. Yu, and J. Paik, Fundamentals of digital image processing. 2018.

[17] C. L. Bai and H. Liu, "Image enhancement based on wavelet transform with MATLA," 2011 Int. Conf. Multimed. Technol. ICMT 2011, pp. 368-370, 2011, doi: 10.1109/ICMT.2011.6001955.

[18] K. Satone, A. Deshmukh, and P. Ulhe, "A review of image compression techniques," Proc. Int. Conf. Electron. Commun. Aerosp. Technol. ICECA 2017, vol. 2017-Janua, pp. 97-101, 2017, doi: 10.1109/ICECA.2017.8203651.

[19] M. A. Aswathy and M. Jagannath, "Performance Analysis of Segmentation Algorithms for the Detection of Breast Cancer," Procedia Comput. Sci., vol. 167, pp. 666-676, 2020, doi: 10.1016/j.procs.2020.03.333. 
[20] A. Krizhevsky and G. E. Hinton, "ImageNet Classification with Deep Convolutional Neural Networks," pp. 1-9.

[21] G. H. Granlund, H. Knutsson, and R. Wilson, "Image enhancement.," Fundam. Comput. Vis., pp. 57-67, 1983.

[22] F. R. Mashrur, "Automatic Identification of Arrhythmia from ECG Using AlexNet Convolutional Neural Network," no. December, pp. 20-22, 2019.

[23] T. Kim, S. C. Suh, H. Kim, J. Kim, and J. Kim, "An Encoding Technique for CNN-based Network Anomaly Detection," Proc. 2018 IEEE Int. Conf. Big Data, Big Data 2018, pp. 2960-2965, 2019, doi: 10.1109/BigData.2018.8622568.

[24] L. Mohammadpour, T. C. Ling, C. S. Liew, and C. Y. Chong, "A Convolutional Neural Network for Network Intrusion Detection System," Proc. Asia-Pacific Adv. Netw., vol. 46, no. 0, pp. 50-55, 2018.

[25] A. Burkov, "Machine Learning."

[26] E. Halim, P. P. Halim, and M. Hebrard, "Artificial Intelligent Models for Breast Cancer Early Detection,” Proc. 2018 Int. Conf. Inf. Manag. Technol. ICIMTech 2018, no. September, pp. 517-521, 2018, doi: 10.1109/ICIMTech.2018.8528140.

[27] M. U. Hoque, T. M. S. Sazzad, A. K. M. A. Farabi, I. Hosen, and M. A. Somi, "An Automated Approach to Detect Breast Cancer Tissue Using Ultrasound Images," 1st Int. Conf. Adv. Sci. Eng. Robot. Technol. 2019, ICASERT 2019, vol. 2019, no. Icasert, pp. 31-34, 2019, doi: 10.1109/ICASERT.2019.8934546.

[28] K. V. Devarapu, S. Murala, and V. Kumar, "Denoising of ultrasound images using curvelet transform," in 2010 The 2nd International Conference on Computer and Automation Engineering (ICCAE), 2010, vol. 3, pp. 447-451.

[29] Y. Ebrahim, M. Ahmed, S. C. Chau, and W. Abdelsalam, "An efficient shape representation and description technique," Proc. - Int. Conf. Image Process. ICIP, vol. 6, pp. 441-444, 2007, doi: 10.1109/ICIP.2007.4379616.

[30] R. Srisha and A. Khan, "Morphological Operations for Image Processing: Understanding and its Applications," NCVSComs-13, no. December, pp. 17-19, 2013. 